\title{
Are eyes special? It depends on how you look at it
}

\author{
JELENA RISTIC \\ University of British Columbia, Vancouver, British Columbia, Canada \\ CHRIS KELLAND FRIESEN \\ University of Calgary, Calgary, Alberta, Canada \\ and \\ ALAN KINGSTONE \\ University of British Columbia, Vancouver, British Columbia, Canada
}

\begin{abstract}
Recent behavioral data have shown that central nonpredictive gaze direction triggers reflexive shifts of attention toward the gazed-at location (e.g., Friesen \& Kingstone, 1998). Friesen and Kingstone suggested that this reflexive orienting effect is unique to biologically relevant stimuli. Three experiments were conducted to test this proposal by comparing the attentional orienting produced by nonpredictive gaze cues (biologically relevant) with the attentional orienting produced by nonpredictive arrow cues (biologically irrelevant). Both types of cues produced reflexive orienting in adults (Experiment 1) and preschoolers (Experiment 2), suggesting that gaze cues are not special. However, Experiment 3 showed that nonpredictive arrows produced reflexive orienting in both hemispheres of a split-brain patient. This contrasts with Kingstone, Friesen, and Gazzaniga's (2000) finding that nonpredictive gaze cues produce reflexive orienting only in the face-processing hemisphere of split-brain patients. Therefore, although nonpredictive eyes and arrows may produce similar behavioral effects, they are not subserved by the same brain systems. Together, these data provide important insight into the nature of the representations of directional stimuli involved in reflexive attentional orienting.
\end{abstract}

In attentional cuing experiments, shorter reaction times (RTs) to a target at a cued location than at a noncued location indicates that people have shifted their attention to the cued location. By varying the time interval between the cue and the target, a temporal profile of the attentional effect can be established (cf. Posner, 1980). With modified versions of this standard paradigm, several recent studies have reported that spatially nonpredictive gaze direction facilitates RT to a target appearing at the gazed-at location (Driver et al., 1999; Friesen \& Kingstone, 1998; Langton \& Bruce, 1999). Specifically, RT for a target at the cued (gazed-at) location is shorter than RT for a target at the noncued location 100-300 msec after onset of the gaze cue, and this effect disappears by $1,000 \mathrm{msec}$ (Friesen \& Kingstone, 1998).

This research was supported by an undergraduate award to J.R. from the Natural Sciences and Engineering Research Council of Canada (NSERC), by graduate awards to C.K.F. by NSERC, the Alberta Heritage Foundation for Medical Research, and the Killam Foundation, and by grants to A.K. from NSERC, the Human Frontier Science Foundation, and the Michael Smith Foundation for Health Research. We thank patient J.W. for his participation, the daycare children who participated in this study, their parents for permitting their participation, and Stephen Langton, Bruce Milliken, and an anonymous reviewer for many insightful comments on a previous version of this research. Correspondence concerning this article should be directed to A. Kingstone, Department of Psychology, University of British Columbia, Vancouver, BC, V6T 1Z4 Canada (e-mail: alan.kingstone@ubc.ca).
Friesen and Kingstone (1998) proposed that this reflexive orienting to gaze direction may represent an attentional process that is unique to biologically relevant stimuli and that, as such, it may be subserved by brain regions specific to the processing of faces and eyes. In support of this hypothesis, Kingstone, Friesen, and Gazzaniga (2000) found that when individuals who have had their cerebral hemispheres surgically disconnected (split-brain patients) were presented with nonpredictive gaze stimuli, only the hemisphere specialized for face processing directed attention reflexively to the gazed-at location.

Remarkably, however, there is no published report directly comparing attentional orienting to spatially nonpredictive gaze cues (biologically relevant) with attentional orienting to spatially nonpredictive symbolic cues, such as arrows (biologically irrelevant). Such a comparison represents a crucial test of the "eyes are special" position. It is possible that this test has not been performed because the conventional wisdom in the field is that spatially nonpredictive central arrow cues will not produce reflexive orienting (cf. Langton, Watt, \& Bruce, 2000, p. 55). This view stems largely from a classic study by Jonides (1981, Experiment 2) that required participants to search a briefly presented array of letters for the target L or R. Before the array appeared, a central arrow cue was flashed momentarily at fixation. The arrow pointed randomly at one of the letter locations. The results indicated that if participants were told to ignore the arrow, orienting to the cued location was absent. This suggests that a nonpredictive arrow cue does not trig- 
ger reflexive attention. However, we will show that this finding does not hold when the task is target detection and the arrow cue remains present (see Tipples, 2002, for a similar result). Thus, it is an open question as to how reflexive orienting to gaze and arrow cues compare.

We addressed this issue in three ways. First, we tested the same adult observers with nonpredictive gaze and arrow cues across a range of cue-target intervals to compare the strength, and the temporal profile, of orienting to biologically relevant and irrelevant directional cues. Second, we tested 4- and 5-year-old children with these same conditions. Given that infants are predisposed to attend to faces and eyes (e.g., Maurer, 1985) and begin to follow gaze direction within the 1st year (e.g., D'Entremont, Hains, \& Muir, 1997), we expected that nonpredictive gaze would produce reflexive orienting in young children. In contrast, given children's more limited experience with arrow stimuli, we expected that nonpredictive arrow cues would produce smaller orienting effects or none at all. Finally, we tested J.W., a split-brain patient, with nonpredictive arrow cues. Would the lateralization found for nonpredictive gaze cues (Kingstone et al., 2000) also occur for nonpredictive arrow cues? If not, the implication is that the cortical brain mechanisms subserving reflexive orienting to biologically irrelevant stimuli are distinct from those subserving reflexive orienting to gaze stimuli.

\section{EXPERIMENT 1}

Friesen and Kingstone's (1998) paradigm was modified to compare the attentional effects of nonpredictive gaze and arrow cues. Because we planned to apply precisely the same paradigm to preschool children in Experiment 2, the targets were pictures of a snowman and a cat.

\section{Method}

Participants. Nineteen psychology undergraduate students from the University of British Columbia participated for course credit.

Apparatus. A 3200c Macintosh Power Book presented stimuli on a 12-in. black-and-white monitor. The participants were seated approximately $57 \mathrm{~cm}$ from the monitor. Target detection RT was measured as the time interval between target onset and pressing the space bar (marked with red tape).

Stimuli. Stimuli and trial sequences are illustrated in Figure 1. All the stimuli were black drawings on a white background. For gaze cues, the central fixation stimulus was a line drawing of a happy face subtending $6^{\circ}$. The face contained two $0.8^{\circ}$ circles representing eyes, a $0.2^{\circ}$ circle centered within the face outline representing a nose, and a curved $2.6^{\circ}$-long line representing a smiling mouth. Black filledin circles representing pupils appeared in the eyes. The pupils were centered vertically in the eyes and just touched the left or the right eye outline. The pupils measured $0.5^{\circ}$, and the distance between the eyes was $1^{\circ}$ when measured from the center of each eye. For arrow cues, the fixation stimulus was a horizontal line centered on the screen, $1.9^{\circ}$ in length. An arrow head and an arrow tail appeared at the ends of the central line, both pointing left or both pointing right. Each of the two lines making up an arrow head or a tail measured $0.5^{\circ}$, and the length of an arrow, from the tip of the arrow head to the ends of the tail, was $2.5^{\circ}$. The two target stimuli were drawings of a snowman and a cat. The cat was $2.5^{\circ}$ wide $\times 3^{\circ}$ high; the snowman was $2.5^{\circ} \times 4^{\circ}$. Targets appeared $5^{\circ}$ to the left or right as measured from the center of the face or arrow to the center of the target.

Design. A trial began by presenting a face with blank eyes or a straight line for $936 \mathrm{msec}$. Then pupils or an arrow appeared. A target appeared on the left or right side of the screen 195, 600, or $1,005 \mathrm{msec}$ after cue onset. The stimuli remained on the screen until

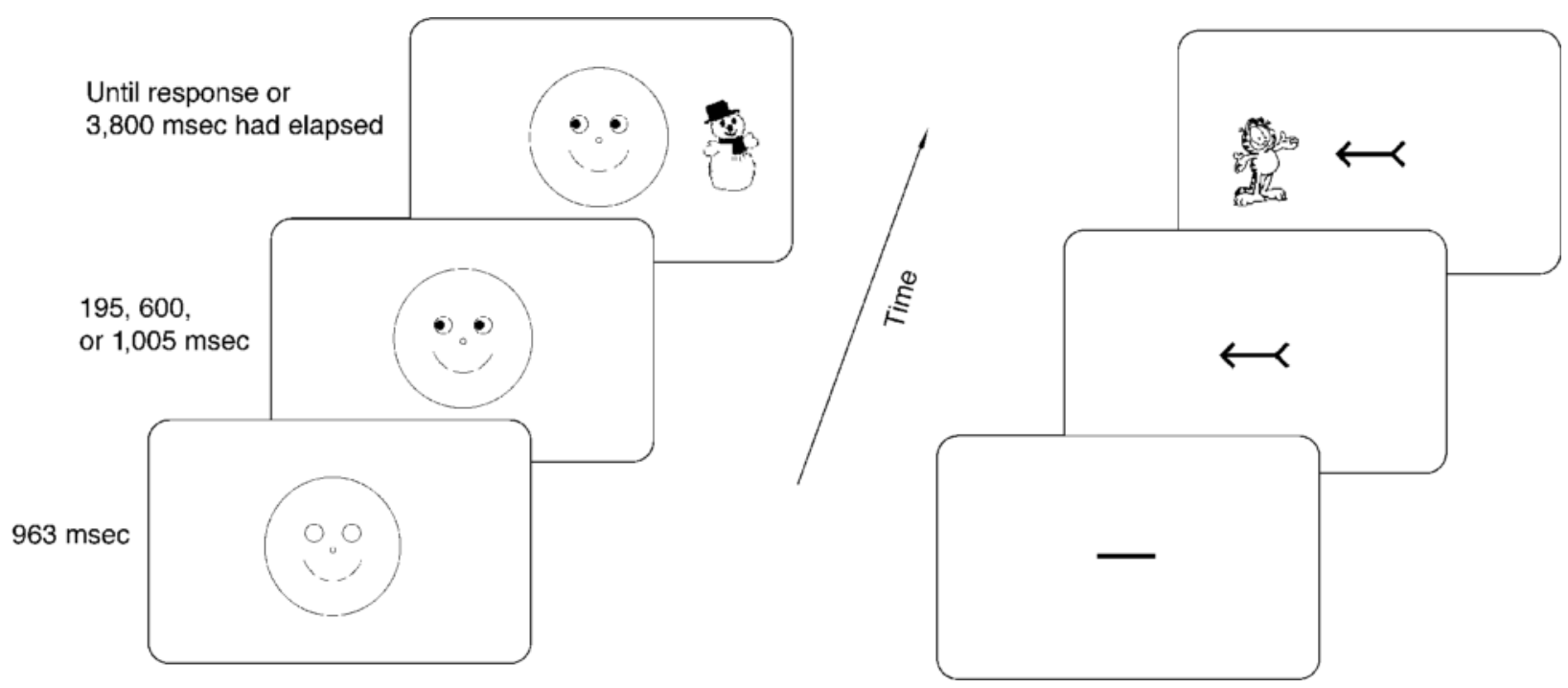

Figure 1. Illustration (not to scale) of stimuli and sample sequence of events for Experiment 1 and Experiment 2 . For both experiments, the start of each trial was signaled by the presentation of either a face with blank eyes or a straight line. The pupils (looking left or right) or an arrow (pointing left or right) appeared $936 \mathrm{msec}$ later. A target (snowman or cat) was presented on the left or the right side of the fixation stimulus 195,600 , or 1,005 msec after cue onset. Both the central cue and the target remained on the screen until a response was made or $3,800 \mathrm{msec}$ had elapsed, whichever came first. Response time was measured from the onset of the target. 
a response was made or 3,800 msec had elapsed, whichever came first. The intertrial interval was $808 \mathrm{msec}$. On cued trials, the target appeared at the location toward which the cue was directed, and on uncued trials, the target appeared at the other location.

The participants completed four blocks of 42 trials, two consecutive blocks with gaze cues and two consecutive blocks with arrow cues. Cue order was counterbalanced across participants (10 received gaze cues first). Cue direction, target location, target identity, and cue-target stimulus onset asynchrony (SOA) were selected randomly and equally. Four catch trials, in which no target was presented, occurred randomly in each block.

Procedure. The participants first received a description of the trial sequence and completed several practice trials. They were told that gaze and arrow direction did not predict target location or identity. Finally, the participants were instructed to press the space bar quickly and accurately when the target appeared and to maintain central fixation during each block.

\section{Results}

Median RTs were calculated for each participant. The interparticipantmeans of these median RTs are illustrated in Figure 2.

Figure 2 shows that for both gaze and arrow cues, RTs were shorter at the cued than at the uncued location at the 195 -msec SOA by approximately the same amount (21

\section{Experiment 1: Adults}
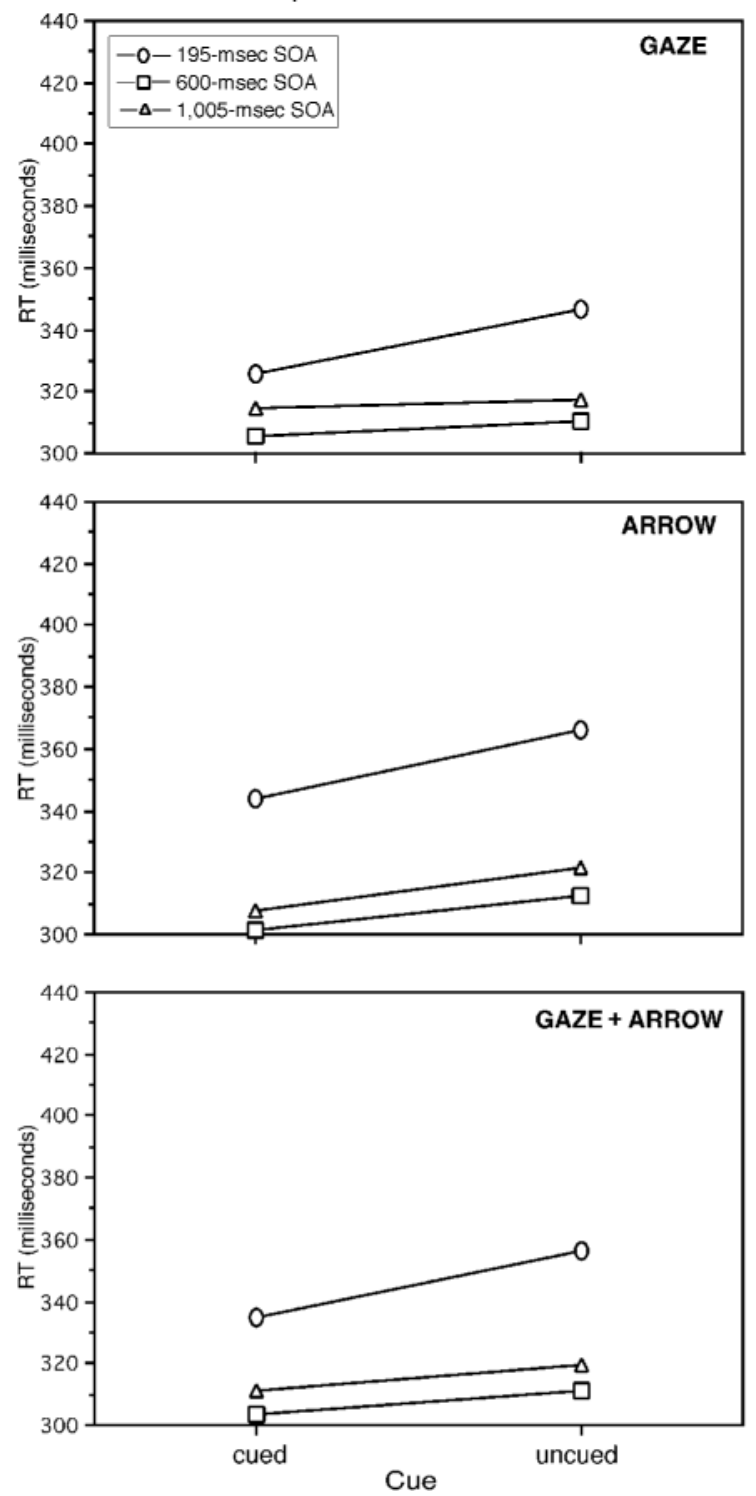

Experiment 2: Children
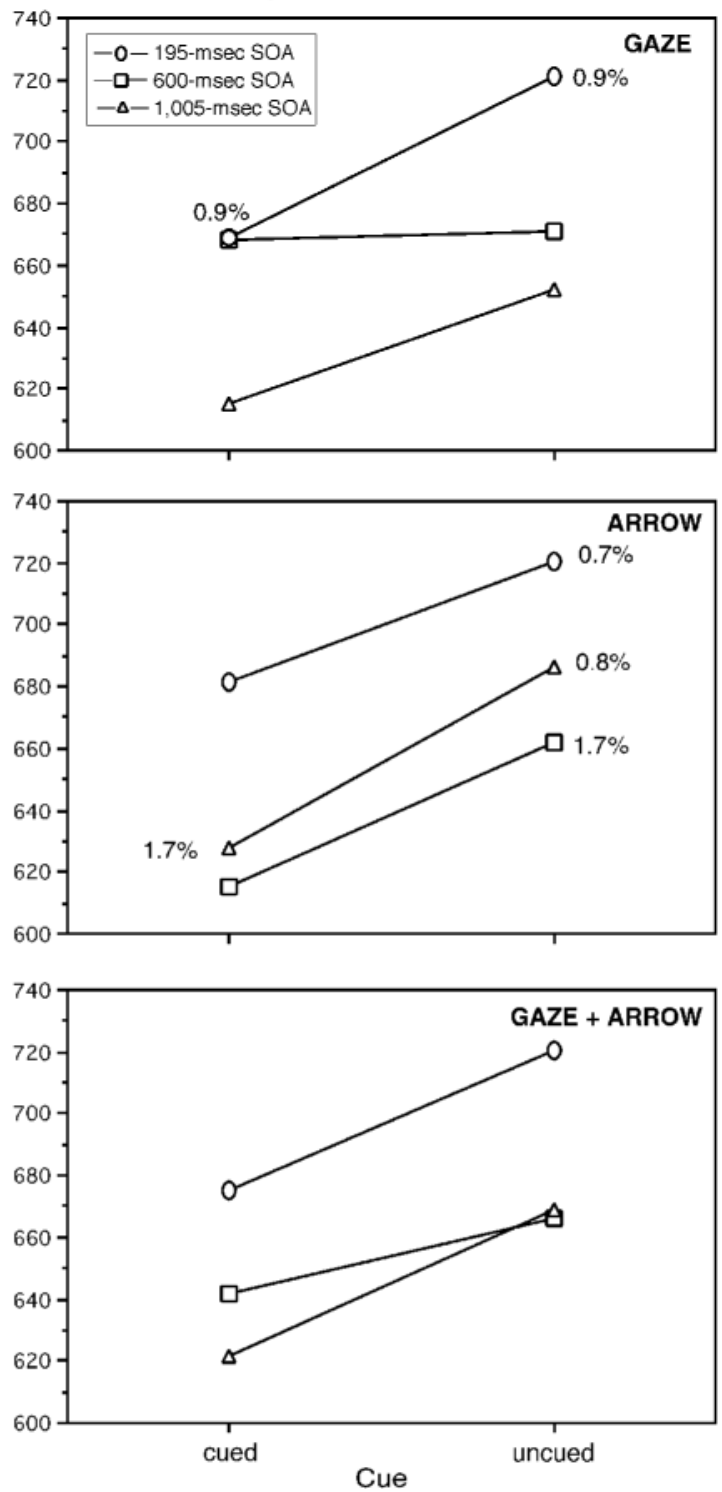

Figure 2. Mean reaction times (RTs, in milliseconds) for Experiment 1 (adult participants) and Experiment 2 (child participants), as a function of cue-target stimulus onset asynchrony (SOA) and cue validity. The top row shows performance for gaze cues, the middle row shows performance for arrow cues, and the bottom row shows performance collapsed across gaze and arrow cue conditions. Error rates $(\%)$ that are not zero are shown. 
and 22 msec, respectively). As SOA lengthened, the RT difference between cued and uncued locations decreased, and RTs became shorter overall (with a slight RT increase at the 1,005-msec SOA, a classic cue-target foreperiod effect; Bertelson, 1967; Mowrer, 1940).

These observations were confirmed by a three-way analysis of variance (ANOVA) with cue type (gaze, arrow), cue validity (cued, uncued), and SOA $(195,600$, and $1,005 \mathrm{msec}$ ) as within-subjects factors. There were significant main effects for validity $[F(1,18)=39.54, p<$ $.0001]$ and SOA $[F(2,18)=40.58, p<.0001]$, reflecting that RTs were shorter at cued than at uncued locations and shorter overall at the longer SOAs. There was also a significant validity $\times$ SOA interaction $[F(2,18)=5.19, p<$ $.02]$, representing that the difference between cued and uncued locations decreased as SOA lengthened. Planned contrasts revealed that the cuing effect was significant at all SOAs (all $F \mathrm{~s}>5$, all $p \mathrm{~s}<.05$ ). Finally, the interaction between cue type and SOA was significant $[F(2,18)=$ $13.93, p<.0001]$, reflecting that RTs were initially longer for an arrow cue than for a gaze cue. No other effects approached significance. In particular, there was no significant main effect for cue type $[F(1,18)=1.36, p>.25]$ and no interaction involving cue type and validity [cue $\times$ validity, $F(1,18)=1.79, p=.20$; cue $\times$ SOA $\times$ validity, $F<1]$.

There were no incorrect keypresses on target-present trials. False alarms were classified as errors and were excluded from the analysis. The false alarm rate on catch trials was $2.4 \%$ in the gaze condition and $3.2 \%$ in the arrow condition. There was no significant effect of cue type $(F<1)$.

\section{Discussion}

There were two key findings in Experiment 1. First, the basic pattern reported by Friesen and Kingstone (1998) and others (Driver et al., 1999; Langton \& Bruce, 1999) was replicated. That is, nonpredictive gaze direction triggered a rapid shift of attention to the gazed-at location. The result was RT facilitation at the gazed-at location $195 \mathrm{msec}$ after onset of the social cue, with the facilitatory effect declining as the cue-target SOA approached $1,000 \mathrm{msec}$.

Second, this effect was observed also for nonpredictive arrow cues. In other words, the reflexive attentional effect is not unique to biologically relevant gaze cues. On this point, the data are absolutely unequivocal. Nonpredictive arrows trigger a reflexive shift of attention to the cued location in a manner that is effectively indistinguishable from gazed-triggered orienting. Indeed, the only difference between the two cues was that, initially, RTs were longer for arrows than for eyes, reflecting perhaps the fact that a gaze is more alerting than an arrow.

Finally, note that Tipples (2002) reported reflexive orienting to peripheral nonpredictive arrow cues. However, as he noted, this effect might be an artifact of the arrow cues' being presented peripherally and/or bilaterally. The present data rule out these possibilities, and as such, to our knowledge, they represent the first clear demonstration that a nonpredictive central arrow cue will trigger reflexive orienting to a cued peripheral location.

\section{EXPERIMENT 2}

In Experiment 2, we tested 3- to 5-year-old preschool children with exactly the same stimulus conditions as the ones that were applied to the adults in Experiment 1. As was noted in the introduction, there is a tremendous amount of evidence indicating that infants are predisposed to preferentially process faces and eyes and that, within their $1 \mathrm{st}$ year, they direct their attention to where others are looking (e.g., D'Entremont et al., 1997; Maurer, 1985). This suggested to us that nonpredictive gaze cues would have greater attentional saliency for children than would biologically irrelevant symbolic stimuli, such as arrows, with which children have less experience. Thus, we predicted that nonpredictive gaze cues would produce orienting effects in preschool children and that nonpredictive arrow cues would produce either smaller orienting effects than nonpredictive gaze cues or no orienting effects at all.

It is also worth noting that a comparison of the adults' and the children's results would provide a novel way to test the extent to which attentional orienting to nonpredictive gaze and arrow cues is reflexive in nature. Specifically, there have been recent suggestions that reflexive orienting to gaze cues in adults may be a learned volitional process (Vecera, 2000). Because children younger than 8 years had demonstrated adultlike reflexive attention effects, but muted volitional attention effects, in peripheral cuing experiments (Brodeur, Trick, \& Enns, 1997), we reasoned that if orienting to biologically relevant (or irrelevant) stimuli was a volitional process, the orienting effects should be smaller for the preschoolers than for the adults.

\section{Method}

Participants. Twenty-eight preschool children were recruited from two Vancouver daycare facilities, and parental permission was obtained for each child. Nine children failed to complete the experiment. Their ages ranged from 3 years, 9 months to 5 years, 10 months (mean age, 4 years, 8 months).

Apparatus, Design, and Procedure. The apparatus, design, and procedure were the same as those in Experiment 1, with two exceptions: (1) Each cue condition was composed of one block of 42 trials, and (2) two experimenters were present when the experiment was conducted. One experimenter ensured that central fixation was maintained, and the other ensured that the correct response key was pressed. Extra care was taken in explaining that the direction of the eyes and arrows did not predict where the target would appear or what target would appear.

\section{Results}

Figure 2 shows that for both gaze and arrow cues, RTs were shorter at the cued than at the uncued location at the $195-\mathrm{msec}$ SOA and that this effect persisted across all SOAs (although disappearing temporarily at the $600-\mathrm{msec}$ 
SOA for gaze cues). As SOA lengthened, RTs became shorter overall (the cue-target foreperiod effect).

Mirroring the adult data in Experiment 1, a three-way ANOVA revealed significant main effects for cue validity $[F(1,18)=18.88, p<.0005]$ and SOA $[F(2,18)=4.24$, $p<.03]$. There was no significant main effect for cue type $(F<.01)$ and no significant interactions (all $F \mathrm{~s}<1.2$, all $p \mathrm{~s}>.31)$.

Incorrect keypresses on target-present trials were $0.3 \%$ for the gaze condition and $0.8 \%$ for the arrow condition, and these did not vary as a function of validity or SOA (all $\left.F_{\mathrm{S}}<1\right)$. The false alarm rate on catch trials was $41.5 \%$ for the gaze condition and $40 \%$ for the arrow condition, with a nonsignificantdifference between cue conditions $(F<1)$. These rates are obviously very high; however, it is important to note that they do not compromise the effects observed on target-present trials, because false alarm responses normally occurred long after the gaze and arrow cues were presented (mean RTs of 1,841 and $1,731 \mathrm{msec}$, respectively), with these long RTs falling well outside the latencies observed on target-present trials. Thus, the high false alarm rates merely reflected the fact that the children had difficulty inhibiting a keypress response for the full duration of a catch trial - that is, they were not target anticipations.

Finally, we performed a four-way ANOVA to compare the adults' and the children's RT data. Cue type, cue validity, and SOA were included as within-subjects variables, and age group (adults vs. children) was included as a between-subjects variable. Consistent with previous findings (e.g., Enns \& Akhtar, 1989), adults' RTs were shorter overall $[F(1,36)=192.68, p<.0001]$. The main effect for cue type was not significant $[F(1,36)=0.050, p>.82]$, and the main effects for SOA $[F(2,36)=11.50, p<.0001]$ and validity $[F(1,36)=31.44, p<.0001]$ were highly significant. The validity $\times$ age group interaction was also significant $[F(1,36)=8.3, p<.007]$, indicating that the children showed a larger cuing effect than did the adults. Of course, whether children would show a larger cuing effect if they were not significantly slower than adults must still be determined. No other interaction was significant (all $F_{\mathrm{s}}<1.6$, all $p \mathrm{~s}>.20$ ).

\section{Discussion}

The results from Experiment 2 are clear-cut. Consistent with our expected finding, the children oriented attention reflexively to the location cued by a nonpredictive gaze stimulus. Unexpectedly, however, the children also oriented attention reflexively to the location cued by a nonpredictive arrow stimulus, and this effect was statistically indistinguishable from the gaze effect. These data replicate for children what we observed for adults in Experiment 1 and again bring into question the notion that the effects of a nonpredictive gaze stimulus are unique to biologically relevant stimuli.

\section{EXPERIMENT 3}

The results of the first two experiments strongly indicate that reflexive orienting to a biologically relevant nonpre- dictive gaze cue has a behavioral effect that is indistinguishable from reflexive orienting to a biologically irrelevant nonpredictive arrow cue. Does this mean that the two types of cues activate the same brain pathways?

A recent study by Kingstone et al. (2000) revealed that split-brain patients shift their attention reflexively to a gazed-at location only when the gaze cue projects to the hemisphere that is specialized for the processing of face stimuli. This suggests that reflexive attention to gaze direction reflects an interaction between neurons in the temporal cortex of the hemisphere specialized for processing faces and eyes and neurons in the parietal cortex responsible for orienting spatial attention (Harries \& Perrett, 1991; Hoffman \& Haxby, 2000; Puce, Allison, Bentin, Gore, \& McCarthy, 1998; Wicker, Michel, Henaff, \& Decety, 1998).

Would a split-brain patient show a similar lateralization of function for nonpredictive arrows? If the neural mechanisms that are responsible for orienting attention to nonpredictive arrow cues are the same as the neural mechanisms responsible for orienting attention reflexively to nonpredictive gaze cues, then J.W. should demonstrate reflexive orienting in the right hemisphere, but not in the left hemisphere.

\section{Method}

Participants. Callosotomy patient J.W. is a 46-year-old male who suffered from intractable epilepsy beginning in 1972. Both hemispheres comprehend language, although verbal and written language output is lateralized to the left hemisphere. This patient has participated in numerous behavioral investigations and is well known for holding central fixation on instruction. See Gazzaniga, Nass, Reeves, and Roberts (1984) for a detailed description of this patient.

Stimuli. The stimuli were controlled by an Apple Macintosh PowerBook 180c computer connected to a 14-in. monitor. The stimuli, illustrated in Figure 3, were black, and the background was white. The arrow stimuli were the same as those in Experiments 1 and 2 and were positioned $2.4^{\circ}$ to the left and right of fixation. The target was an asterisk that subtended $0.7^{\circ}$ and always appeared $4.2^{\circ}$ away from the central fixation cross (which subtended $0.3^{\circ}$ ).

Procedure. J.W. was centered with respect to the screen and keyboard, and central fixation was held without difficulty throughout each block of trials. Twenty practice trials preceded two sets of 10 blocks of 64 test trials, for a total of 1,280 test trials. J.W. was informed repeatedly, and understood, that arrow direction did not predict where the target would appear. He was strongly encouraged to respond as quickly and as accurately as he could, by pressing a lefthand key $(\mathrm{Z})$ when the target was presented to the left visual field (LVF; right hemisphere) and a right-hand key (/) when the target was presented to the right visual field (RVF; left hemisphere).

Figure 3 presents an example sequence of events for a trial. Two vertical lines were presented concurrently to the left and to the right of fixation. Arrow heads and tails appeared $675 \mathrm{msec}$ later, creating arrows that pointed up or down. After 105 or $600 \mathrm{msec}$, a target appeared above or below one of the arrows. Arrow direction, target location, and cue-target SOA were selected randomly and equally within each block. The stimuli remained on the screen until a response was made or 2,700 msec had elapsed, whichever came first. The intertrial interval was $675 \mathrm{msec}$. Note that this procedure duplicated that in Kingstone et al. (2000) except that arrow cues replaced gaze cues.

\section{Results}

Figure 3 shows that for both LVF (right hemisphere) and RVF (left hemisphere) targets, RTs were shorter at the 

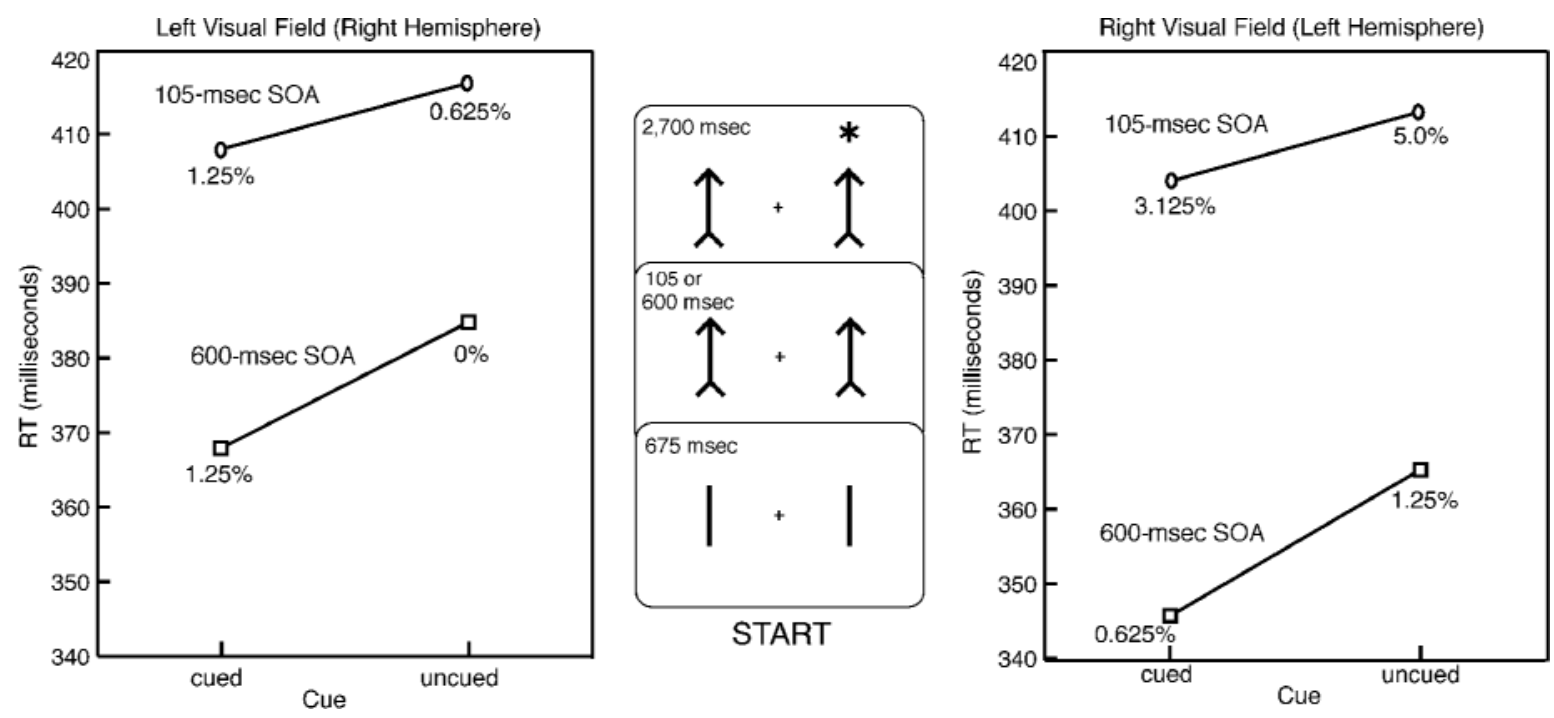

Figure 3. Illustration (not to scale) of stimuli, sample sequence of events, and mean response time (RT) for Experiment 3 (splitbrain participant J.W.) as a function of cue-target stimulus onset asynchrony (SOA), cue validity, and visual field. The error rates $(\%)$ for all conditions are shown. See the text for procedural details.

cued than at the uncued location at 105- and 600-msec SOAs. In addition, as SOA increased, RTs became shorter overall (the cue-target foreperiod effect), with this effect being greater for the LVF than for the RVF targets. These arrow cue data contrast dramatically with Kingstone et al.'s (2000) finding that gaze direction triggered reflexive orienting only for the LVF (right hemisphere) targets.

RT and accuracy data were subjected to an ANOVA, with cue validity, SOA, and target field as factors. RT analysis revealed that all main effects were significant (all $F_{\mathrm{S}}>8.93$, all $\left.p \mathrm{~s}<.01\right)$. There was also a significant interaction between SOA and target field $[F(1,1251)=5.38$, $p<.02$ ], reflecting the greater foreperiod effect for LVF than for RVF targets. Importantly, there were no other significant interactions (all $F \mathrm{~s}<1.5$, all $p \mathrm{~s}>.20$ ). Error analysis produced no significant effects (all $F_{\mathrm{S}}<1.2$, all ps $>.35)$.

\section{Discussion}

The finding that nonpredictive arrow direction produces a cuing effect in both hemispheres of split-brain patient J.W., at both SOAs, contrasts with Kingstone et al.'s (2000) finding that nonpredictive gaze direction produces a rapid and short-lived attention effect that is lateralized to J.W.'s face/gaze-processing right hemisphere. Together these two findings strongly suggest that the neural mechanisms that subserve a reflexive shift of attention in response to nonpredictive gaze direction are fundamentally different from the mechanisms that subserve reflexive orienting in response to nonpredictive arrows. This agrees with current work indicating that there exists a distinct brain region that is specialized for processing biologically relevant directional face and gaze information, which is not activated by inanimate biologically irrelevant directional information, such as arrows (see Kanwisher, Downing, Epstein, \& Kourtzi, 2001, for a review).

\section{GENERAL DISCUSSION}

Three experiments were conducted to examine whether attentional orienting triggered by spatially nonpredictive and biologically relevant gaze cues differs from attentional orienting triggered by spatially nonpredictive and biologicallyirrelevant cues, such as arrows. The results were unambiguous.

Our first two experiments showed that the behavioral effects of nonpredictive gaze cues and arrow cues were significant and equivalent across adults and children. At first pass, these data would appear to compromise the "eyes are special" position put forward by Friesen and Kingstone (1998), Langton and Bruce (1999), and Driver et al. (1999). That is, the position that the reflexive attentional orienting observed for gaze cues reflects an attentional network that is qualitatively distinct from attentional orienting triggered by biologically irrelevant stimuli.

In support of this position, Kingstone et al. (2000) found that when nonpredictive gaze cues are presented to split-brain patient J. W., only the right (face/gaze-processing) hemisphere attends reflexively to the gazed-at location. Although it is tempting to conclude that this effect is specific to the processing of biologically relevant face stimuli, an alternative possibility is that any directional stimulus, biologically relevant or not, will trigger a lateralized reflexive shift of attention (see Hommel, Pratt, Colzato, \& Godijn, 2001, for a similar consideration with regard to nonpredictive arrows and words). Indeed, the behav- 
ioral equivalence of gaze and arrows observed in Experiments 1 and 2 supports this alternative. It is, therefore, new and significant to discover in Experiment 3 that nonpredictive arrows produce reflexive orienting in both hemispheres of split-brain patient J.W., in contrast to the lateralized effect obtained with nonpredictive gaze. In this very important sense, biologically relevant gaze cues are special.

Note that the split-brain data do not indicate simply that any index of attentional orienting to gaze direction will be lateralized to the hemisphere that is preferentially biased to the processing of face and gaze information. Rather, the key is whether the attentional orienting is reflexive (triggered by a nonpredictive gaze cue) or volitional (triggered by a predictive cue; see Danziger \& Kingstone, 1999, for a recent review of exogenous vs. endogenous orienting). To demonstrate this point, Kingstone et al. (2000) conducted a control study wherein they repeated the procedure used in the present Experiment 3, but with gaze stimuli that predicted where the target stimulus was likely to appear-that is, in either visual field, the target appeared at a gazed-at location on $75 \%$ of the trials and at a nongazed-at location on $25 \%$ of the trials. Here, both hemispheres attended volitionally to the predicted gazed-at location. Thus, the lateralization of reflexive attention to nonpredictive gaze direction reflects an interaction between gaze processing and the predictive (attentional) value of the gaze cue.

In sum, our results with J.W. (present study; Kingstone et al., 2000) indicate that reflexive orienting to nonpredictive gaze is subserved by a neural system that is qualitatively different both from the system that supports reflexive attention to biologically irrelevant stimuli and from the system that supports volitional orienting to predictive gaze direction. In this important way, reflexive orienting to eye direction is special. Moreover, a recent study with healthy adult observers indicates that reflexive and volitional orienting to gaze direction are behaviorally separable. Friesen, Ristic, and Kingstone (2002) discovered that if eye direction predicts that a target will appear at a non-gazed-at location, attention is committed reflexively to the gazed-at location concurrently with volitonal orienting to the predicted location. Counterpredictive arrows, on the other hand, appear to produce only reflexive or volitional effects. Thus, we find that even in healthy observers, behavioral differences between eyes and arrows may emerge, consistent with the finding of the present study that eyes are indeed special.

\section{REFERENCES}

Bertelson, P. (1967). The time course of preparation. Quarterly Journal of Experimental Psychology, 19, 272-279.

Brodeur, A. D., Trick, M. I., \& ENNs, J. T. (1997). Selective attention over the lifespan. In A. J. Burack \& J. T. Enns (Eds.), Attention, development, and psychopathology (pp. 74-97). New York: Guilford.
DAnziger, S., \& Kingstone, A. (1999). Unmasking the inhibition of return phenomenon. Perception \& Psychophysics, 61, 1024-1037.

D'Entremont, B., Hains, S. M. J., \& Muir, D. W. (1997). A demonstration of gaze following in 3- to 6-month-olds. Infant Behavior \& Development, 20, 569-572.

Driver, J., Davis, G., Ricciardelli, P., Kidd, P., Maxwell, E., \& BARON-CoHEN, S. (1999). Gaze perception triggers visuospatial orienting by adults in a reflexive manner. Visual Cognition, 6, 509-540.

ENNS, J. T., \& AKhTAR, N. (1989). Relations between covert orienting and filtering in the development of visual attention. Journal of Experimental Child Psychology, 48, 315-334.

Friesen, C. K., \& Kingstone, A. (1998). The eyes have it! Reflexive orienting is triggered by nonpredictive gaze. Psychonomic Bulletin \& Review, 5, 490-495.

Friesen C. K., Ristic, J., \& Kingstone, A. (2002). Reflexive and volitional orienting to directional cues: Separable attention effects unique to biologically relevant gaze stimuli. Manuscript submitted for publication.

Gazzaniga, M. S., Nass, R., Reeves, A., \& Roberts, D. (1984). Neurologic perspectives on right hemisphere language following surgical section of corpus callosum. Seminars in Neurology, 13, 536-540.

Harries, M., \& Perrett, D. I. (1991). Visual processing of faces in temporal cortex: Physiological evidence for a modular organization and possible anatomical correlates. Journal of Cognitive Neuroscience, 3, 9-24.

Hoffman, E. A., \& Haxby, J. V. (2000). Distinct representations of eye gaze and identity in the distributed human neural system for face perception. Nature Neuroscience, 3, 80-84.

Hommel, B., Pratt, J., Colzato, L., \& Godisn, R. (2001). Symbolic control of visual attention. Psychological Science, 12, 360-365.

JoNIDES, J. (1981). Voluntary vs. automatic control over the mind's eye's movement. In J. B. Long \& A. D. Baddeley (Eds.), Attention and performance IX (pp. 187-203). Hillsdale, NJ: Erlbaum.

Kanwisher, N., Downing, P., Epstein, R., \& Kourtzi, Z (2001). Functional neuroimaging of visual recognition. In R. Cabeza \& A. Kingstone (Eds.), Handbook of functional neuroimaging of cognition (pp. 109-151). Cambridge, MA: MIT Press.

Kingstone, A., Friesen, C. K., \& Gazzaniga, M. S. (2000). Reflexive joint attention depends on lateralized cortical connections. Psychological Science, 11, 159-165.

Langton, S. R. H., \& BRUCE, V. (1999). Reflexive social orienting. Visual Cognition, 6, 541-567.

Langton, S. R. H., Watt, R. J., \& BRuce, V. (2000). Do the eyes have it? Cues to the direction of social attention. Trends in Cognitive Sciences, 4, 50-59.

Maurer, D. (1985). Infants' perception of facedness. In T. M. Field \& N. A. Fox (Eds.), Social perception in infants (pp. 73-100). Norwood, NJ: Ablex.

Mowrer, O. H. (1940). Preparatory set (Expectancy): Some methods of measurements. Psychological Review Monograph, 52 (Whole No. 233).

Posner, M. I. (1980). Orienting of attention. Quarterly Journal of Experimental Psychology, 32, 3-25.

Puce, A., Allison, T., Bentin, S., Gore, J. C., \& McCarthy, G. (1998). Temporal cortex activation in humans viewing eye and mouth movements. Journal of Neuroscience, 18, 2188-2199.

TiPplES, J. (2002). Eye gaze is not unique: Automatic orienting in response to uninformative arrows. Psychonomic Bulletin \& Review, 9, 314-318.

VECERA, S. (2000). The eyes may not have it: Interrupting the allocation of "social attention." Unpublished manuscript.

Wicker, B. F., Michel, F., Henaff, M., \& Decety, J. (1998). Brain regions involved in the perception of gaze: A PET study. NeuroImage, 8, 221-227.

(Manuscript received April 10,2001; revision accepted for publication October 19, 2001.) 